\title{
The Role of Amorphous Precursor in Phase Selection Hierarchy in Marginal Metallic Glasses
}

\author{
C. Yildirim ${ }^{1}$, M. Kutsal ${ }^{1}$, R.T. Ott ${ }^{2}$, M.F. Besser ${ }^{2}$, M. J. Kramer ${ }^{2}$ and Y. E. Kalay ${ }^{1,{ }^{*}}$ \\ ${ }^{1}$ Department of Metallurgical and Materials Engineering, METU, Ankara, 06800 Turkey \\ ${ }^{2}$ Ames Laboratory (DOE), Ames, IA 50011, USA
}

\begin{abstract}
The solid state amorphous structure and devitrification products of $A l_{90} T b_{10}$ alloys produced by Cu block melt-spinning and magnetron sputtering were investigated by a combined study of differential scanning calorimetry, in-situ $X$-ray diffraction and conventional transmission electron microscopy. The as-prepared specimens were found to be fully amorphous according to the electron and X-ray diffraction. The thermal analysis and in-situ XRD analyses of the amorphous samples having the same chemistry but different processing history showed clear differences in the devitrification pathways in terms of nanocrystal population, size and the phase selection. The variations in the crystallization path were explained on the basis of distinct topological and chemical medium range ordering observed in as-quenched and as-sputtered amorphous structures.
\end{abstract}

Keywords: Phase selection, medium-range order, nanocrystallization, marginal metallic glass

Corresponding author. Tel: +90 312210 2525; fax: +90 3122102518

E-mail address: ekalay@metu.edu.tr (Y.E. Kalay) 


\section{Introduction}

Aluminum based marginal glass-forming alloys, Al-RE, Al-TM-RE (RE: rare-earth element; TM: transition metals), comprise an important family of metallic glasses due to their ability of forming of high number density of fcc-Al nanocrystals upon devitrification [1-3]. The high-density of fcc-Al nanocrystals embedded in amorphous matrix can lead to significant increase in mechanical strength in partially devitrified condition [4-8]. As marginal glassformers need higher cooling rates as compared to conventional metallic glasses, much higher cooling rates on the order of $10^{5}-10^{6} \mathrm{~K} / \mathrm{s}$ is required. Among vast varieties of techniques, meltspinning and magnetron sputtering are well established processes to reach higher cooling rates. In melt-spinning process, the molten metal is ejected onto a rotating copper wheel [9] on the other hand in magnetron sputtering the metal is coated onto a cooled Si substrate by the bombardments of ions to the target surface [10]. As its name implies, in melt-spinning glass solidifies from metallic melt, yet in magnetron sputtering glass forms via condensation of the metallic vapor.

Although these systems have been extensively studied, a complete explanation of the underlying nucleation mechanism resulting in a nanocomposite structure with $10^{21-24} \mathrm{~m}^{-3}$ nuclei embedded in an amorphous matrix is still lacking. Several hypothesis, such as "quenched-in nuclei" [11,12], "phase separation in amorphous state" [13] have been previously proposed, however they do not explain the totality of the experimental observations. A more recent study on the short to medium-range ordering offers a different perspective by taking into account the topological ordering in the liquid state in Al-Tb system [14]. It was reported that high number density of Al clusters in as-quenched states are inherited from the liquid state prior to quenching the melt, caused by Tb atoms attracting Al 
atoms in the first shell neighborhood, thus forming a continuous network. Upon rapid solidification, these chemically isolated regions are retained in the amorphous matrix with isolated regions of nearly pure Al, which act as nucleating sites for fcc-Al during devitrification [14]. In another recent study, the existence of such nanoscale amorphous regions, although larger in size, was also confirmed by atom probe tomography (APT) in Al-Y-Fe [15].

In this study we report the role of the short-to-medium range atomic order of the amorphous precursor structure on devitrification behavior of $\mathrm{Al}_{90} \mathrm{~Tb}_{10}$ amorphous alloys. The amorphous and partially crystallized states have been investigated for samples that were produced by two different processes, namely melt spinning and magnetron sputtering. Differences in the thermal devitrification behavior for glasses of same overall composition have been characterized with differential scanning calorimetry (DSC), in-situ x-ray diffraction and transmission electron microscopy (TEM).

\section{Experimental Procedure}

Amorphous ribbons of $\mathrm{Al}_{90} \mathrm{~Tb}_{10}$ with 25 micrometer thickness and $1.2 \mathrm{~mm}$ width were produced by a single copper block melt spinner, quenching from $1423 \mathrm{~K}$ at a tangential wheel speed of $30 \mathrm{~m} / \mathrm{s}$. The ingots were prepared in a copper-hearth electric arc melting in an argon atmosphere and re-melted three times to ensure compositional homogeneity. Amorphous $\mathrm{Al}_{90} \mathrm{~Tb}_{10}$ thin films with 10 micrometer thickness were produced by DC magnetron sputtering onto a liquid nitrogen cooled Si wafer. The chemical compositions of as-quenched and assputtered alloys were confirmed by electron spectroscopy. EDS indicated 90.3 and 90.6 at\% aluminum for the melt-spun ribbon (as an average of wheel and air sides) and sputtered thin films, respectively. Differential scanning calorimetry (DSC) experiments were conducted using SEIKO SII X-DSC7000. In DSC experiments, approximately 7 to $10 \mathrm{mg}$ 's of ribbon or sputtered thin- 
films were filled in Al pans and covered with Al lids, and heated-up from room temperature to $600 \mathrm{~K}$ at 10 and $40 \mathrm{~K} / \mathrm{min}$ under a protective $\mathrm{N}_{2}$ gas atmosphere. After each scan, an empty pan was placed into the instrument for baseline correction. The isochronal annealing for transmission electron microscopy (TEM) investigations were performed using the same calorimeter with the identical experimental conditions in which DSC traces were obtained. Conventional TEM analyses were conducted using JEOL JEM2100F (at METU) field emission STEM at $200 \mathrm{keV}$. Specimens for TEM analyses were prepared using electropolishing with a solution of 25 vol.\% nitric acid and 75 vol.\% methanol at $241 \mathrm{~K}$. In-situ X-ray diffraction experiments were carried out at BL04-MSPD beamline of ALBA Synchrotron Light Laboratory. A double Si monochromator was used to select the wavelength of $0.04136 \mathrm{~nm}$. For room temperature experiments, free-standing samples were attached such that the incoming beam hit the sample surface, inclined with $45^{\circ}$. The diffraction data were collected in the Q-range $(Q=4 \pi \sin (\theta) / \lambda)$ where $\theta$ is the Bragg's angle and $\lambda$ is the wavelength of X-ray radiation) of 8.3$75 \mathrm{~nm}^{-1}$ using a Mythen array detector system. Melt-spun ribbons and sputtered films were cut by a razor blade and filled into X-ray transparent capillaries having $0.5 \mathrm{~mm}$ diameter and $10 \mu \mathrm{m}$ wall thicknesses. The capillaries were heated from room temperature to $633 \mathrm{~K}$ for the sputtered thin-films and to $773 \mathrm{~K}$ for the melt-spun ribbons using a hot air blower placed beneath at a constant heating rate of $10 \mathrm{~K} / \mathrm{min}$. The temperature of the samples was measured using the thermocouples placed right before the exit of the blower having $\pm 2 \mathrm{~K}$ tolerance. Capillaries were heated continuously with the given heating rate and diffraction data were collected in every 3 seconds within the Q-range of 5.3- $65.8 \mathrm{~nm}^{-1}$. Intensity versus Bragg angle data of both samples were first were corrected for background, polarization, absorption, multiple, Compton scattering and then converted to total X-ray structure factor using pdfgetX3 software [16]. 


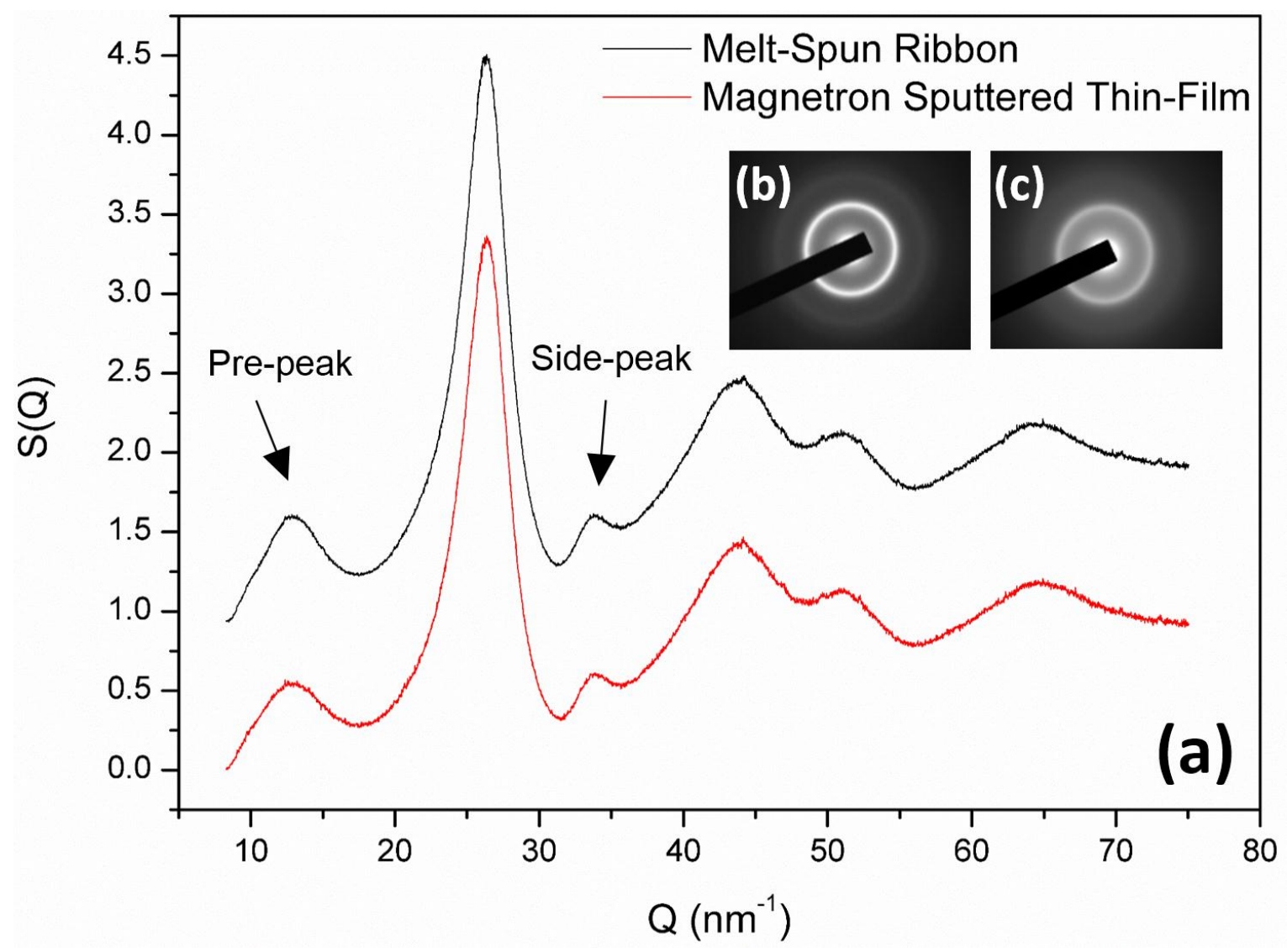

Figure 1. (a) Room temperature total X-ray structure factors $(S(Q))$ of melt-spun ribbons (black) and sputtered thin-films (red) of $\mathrm{Al}_{90} \mathrm{~Tb}_{10}$. (b) and (c) SAD patterns of melt-spun ribbons and sputtered thin-films of $\mathrm{Al}_{90} \mathrm{~Tb}_{10}$ at room temperature, respectively. (For interpretation of the references in color in this figure legend, the reader is referred to the web version of this article).

Table 1. Pre-peak, correlation length, side-peak, and main amorphous peak values of total structure factor $(\mathrm{S}(\mathrm{Q})$. Pre-peak and main amorphous peak were calculated using a Lorentzian fit.

\begin{tabular}{|l|l|l|l|l|}
\hline \multicolumn{1}{|c|}{ Al$_{90}$ Tb $_{10}$} & \multicolumn{1}{|c|}{$\begin{array}{c}\text { Pre-peak } \\
\left(\mathbf{n m}^{-1}\right)\end{array}$} & $\begin{array}{c}\text { Pre-peak } \\
\text { FWHM (rad) }\end{array}$ & \multicolumn{1}{|c|}{$\begin{array}{c}\text { Side-peak } \\
\left(\mathbf{n m}^{-1}\right)\end{array}$} & $\begin{array}{c}\text { Main } \\
\text { Amorphous } \\
\text { Peak (nm }\end{array}$ \\
\hline Melt-spun Ribbon & $13.0(3)$ & $0.09(9)$ & $33.9(1)$ & $26.1(3)$ \\
\hline Sputtered Thin-Film & $12.8(1)$ & $0.13(3)$ & $33.4(4)$ & $26.1(1)$ \\
\hline
\end{tabular}




\section{Results and Discussion}

Figure 1 (a) shows the total X-ray structure factors $(S(Q))$ collected at room temperature for the melt-spun and sputtered samples. The amorphous nature of the asquenched ribbon and as-sputtered thin-film is clearly observed. There are strong pre-peak and side-peak reflections at wave momentum of approximately 13 and $34 \mathrm{~nm}^{-1}$ in ribbon specimens. The existence of such additional reflections with relatively robust intensities was previously associated to RE-Al interactions $[14,17,18]$. The strong atomic coupling between the $\mathrm{RE}$ and $\mathrm{Al}$ atom results in a selective accumulation of $\mathrm{Al}$ atoms around the $\mathrm{Tb}$, which persists in molten state [19]. The average measured coordination number around Tb atoms was found to be 15 using ab-initio constrained RMC simulations, which approximately corresponds to $91 \% \mathrm{Al}$ in the first shell neighborhood [14]. The presence of $\mathrm{Tb}$ atoms with such a high coordination of Al causes the amorphous matrix to be divided into Al-rich and Aldepleted regions. These Al-rich regions were found to be the origin of highly populated fcc-Al nanocrystallization upon devitrification whereas; Al-depleted regions inhibit long-range diffusion and restrict the size of individual fcc-Al nanocrystals to stay less than $50 \mathrm{~nm}$. The selected-area diffraction (SAD) patterns collected from the melt-spun ribbons and the sputtered thin-films of $\mathrm{Al}_{90} \mathrm{~Tb}_{10}$ indicate a fully amorphous structure (Figure 1 (b) and (c)). The total X-ray structure factors, on the other hand, reveal similar pre-peak and side-peak formation at almost exactly the same position with relatively low intensities, as shown in Figure 1 (a). The full-width at half-maximum values calculated by Lorentzian approximation indicated a broader peak for the magnetron sputtered thin-films. The structure related values are tabulated in Table 1 . Similar trend was previously observed between the melt-spun $\mathrm{Al}_{90} \mathrm{~Tb}_{10}$ ribbon and its molten state [14]. The existence of such pre-peak was associated with the existence of highly-populated Al-RE interactions $[10,13-15,17]$. The increase in such 
interactions drives the system a pseudo phase separation into Al-rich and Al-depleted regions $[14,19,21]$. Therefore, although the pre-peak belonging to the sputtered thin-film is much broadened, it may still be the indication of chemically separated regions due to intense Al-RE coupling in a much finer length scale.

The most intriguing observations came with the differential scanning calorimetry analyses of the amorphous as-sputtered thin-films and melt-spun ribbons having the same chemical composition. Figure 2 shows the continuous heating signals of ribbons and thin-films with heating rates of 10 and $40 \mathrm{~K} / \mathrm{min}$. The devitrification paths for the amorphous structure having the same chemistry yet different synthesis are completely dissimilar. The as-spun ribbons have four exothermic events under 10 and $40 \mathrm{~K} / \mathrm{min}$. The exothermic event called as $\mathrm{R} 1$ is known as fcc-Al nanocrystallization from the previous studies $[22,23]$. The exothermic event indicated as R3 is almost overlapped with $\mathrm{R} 2$ for $10 \mathrm{~K} / \mathrm{min}$ heating rate, which is quite apparent in $40 \mathrm{~K} / \mathrm{min}$ heating rate scan. Whether or not R3 represents an individual exothermic event is controversial. R2 may be an asymmetrical peak with R3 acting as a tail, in agreement with what was reported for the fcc-Al nanocrystallization events of $\mathrm{Al}_{88} \mathrm{Y}_{7} \mathrm{Fe}_{5}$ metallic glass [22]. DSC reveals two major exothermic events for the as-sputtered thin-films as called as S1 and S2 between room temperature and $600 \mathrm{~K}$. S1 (inset of Figure 2), indicating the fcc-Al nanocrystallization, has an extremely broad signal that it is difficult to resolve at 10 and $40 \mathrm{~K} / \mathrm{min}$ heating rates. The peak temperatures (halfway through the crystallization) of fcc-Al nanocrystallization in the melt-spun and the sputtered thin-films were found to be located at 521 and $507 \mathrm{~K}$ with $40 \mathrm{~K} / \mathrm{min}$ heating rate, respectively. 


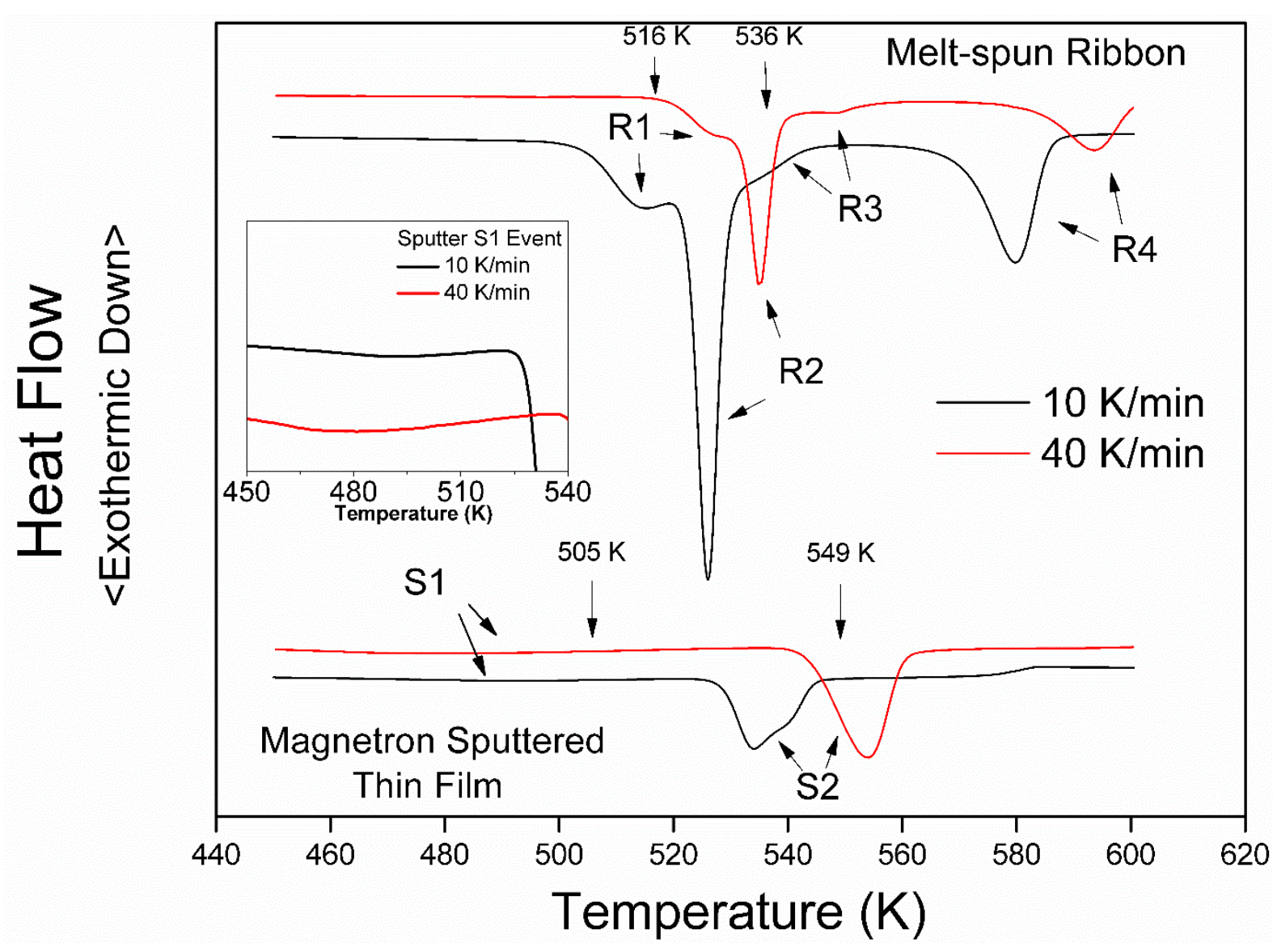

Figure 2. Continuous DSC traces for melt-spun ribbons and sputtered thin-films of $\mathrm{Al}_{90} \mathrm{~Tb}_{10}$ collected at 10 (black) and 40 (red) $\mathrm{K} / \mathrm{min}$ heating rates, respectively. Inset shows the close up of $\mathrm{S} 1$ exothermic event, for clarity. (For interpretation of the references in color in this figure legend, the reader is referred to the web version of this article).

It is clearly observed that the exothermic peaks indicating the crystallization events on DSC patterns possess dissimilar peak temperatures and shapes. In other words, by looking at the phase transformations that take place during continuous heating, one can conclude that the melt-spun ribbons and the sputtered thin-films follow different initial devitrification pathways yet converge at higher temperatures. In-situ XRD is in good agreement with DSC results in terms of presenting the difference in phase selection between amorphous melt-spun ribbons and sputtered thin-films. Figure 3 exhibits the in-situ XRD experiments collected along isochronal heating of $10 \mathrm{~K} / \mathrm{min}$ in $2 \mathrm{D}$ image surfaces, which were produced by integrating the XRD patterns after each interrupted temperature. The lines on the images indicate the location of Bragg peaks in Q-space, with the grey-scale contrast level being proportional to 
diffracted beam intensities. In both as-quenched and as-sputtered precursors, the initial crystallization product was found to be the fcc-Al. Therefore, the S1 and R1 labeled DSC traces should correspond to the formation of nanocrystal aluminum phase. The fcc-Al crystallization in sputtered film occurs at lower temperatures as compared to the ribbon specimens. The second crystallization event of the melt-spun ribbon and the sputtered thin-film is completely different from one another according to the in-situ XRD experiments. The remaining amorphous phase within the melt-spun ribbon specimen transforms into a metastable hexagonal phase with calculated lattice parameters of $a=0.543 \mathrm{~nm}$ and $c=1.773 \mathrm{~nm}$. This phase is missing during the devitrification of sputtered thin-films. Instead, the residual amorphous phase after the fcc-Al crystallization transforms into a metastable cubic phase with a lattice parameter of $a=0.990 \mathrm{~nm}$. This should correspond to S2 crystallization event in DSC. The metastable hexagonal phase in ribbon specimens was found to transform into a similar metastable cubic phase with an elevated onset crystallization temperature. By looking at the sequence of the phase transformations, R4 can be labelled as metastable hexagonal to a metastable cubic phase transformation. Given that there is no phase transformation that had taken place between the metastable hexagonal-to-cubic phases, the exothermic peaks labeled as $\mathrm{R} 3$ can be identified as the asymmetric high temperature tail of the metastable hexagonal phase precipitation. All the high temperature metastable intermetallic phases are accompanied with fcc-Al according to in-situ XRD analyses. The Cu-Ka XRD analysis of the heattreated specimens at $500{ }^{\circ} \mathrm{C}$ for 5 hours indicated the formation stable $\mathrm{Al}_{3} \mathrm{~Tb}$ and $\mathrm{Al}$ phases for both melt-spun and sputtered specimens. 


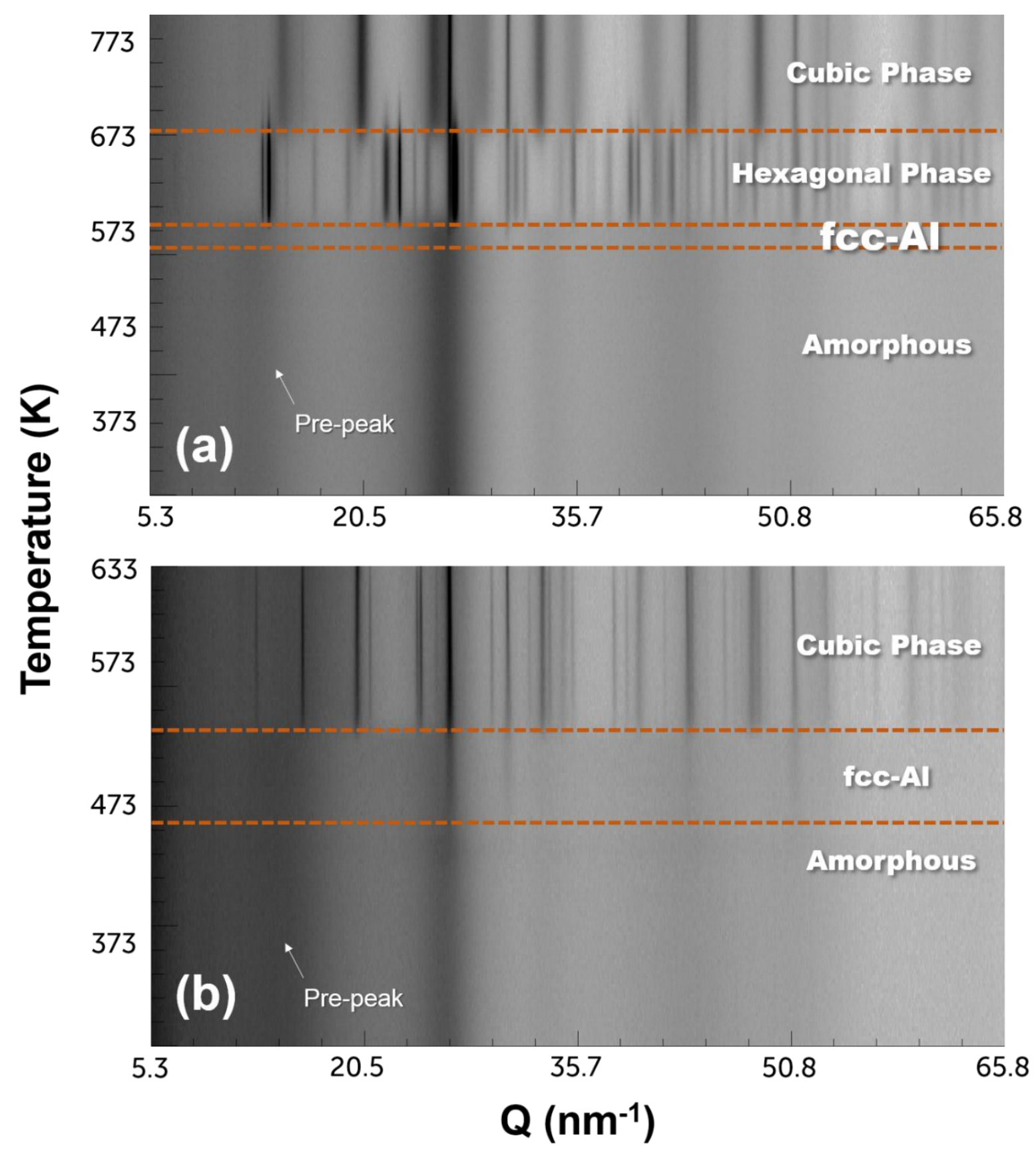

Figure 3. 2D area plots, representing the in-situ XRD results of (a) melt-spun ribbons and (b) magnetron sputtered thin-films of $\mathrm{Al}_{90} \mathrm{~Tb}_{10}$ heated up to $773 \mathrm{~K}$ and $633 \mathrm{~K}$, respectively. It should be noted that the phase name implies the first forming crystal structure.

The DSC and in-situ XRD analyses were supported with interrupted quenched TEM experiments. Specimens prepared for TEM were continuously heated (at $40 \mathrm{~K} / \mathrm{min}$ ) up to critical predetermined temperatures as shown in Figure 2 (indicated by vertical arrows) and 
then quenched to room temperature for TEM investigation. Figure 4 (a) and (b) shows the BF images of the melt-spun ribbons and the sputtered thin-films after initial crystallization event. Melt-spun ribbons show fcc-Al nanocrystals of 30-50 nm average size with $\sim 10^{21} \mathrm{~m}^{-3}$ number of nucleation densities. This is very typical for Al-RE type metallic glasses and such numbers have been reported by a number of studies $[10,18-20]$. The first crystallization product of the sputtered thin-films is also fcc-Al, as predicted by in-situ XRD. However, the morphology, the average size, and the nucleation density are substantially different. The average size is between 2-10 nm and the number of nucleation density reaches up to $10^{24} \mathrm{~m}^{-3}$. The morphology of the fcc-Al nanocrystals has become spherical as compared to their dendritic shaped counterparts in the melt-spun ribbons. The increase in the number of density can be also recognized by the corresponding SAD patterns in Figure 4 (a) and (b). The Bragg reflection rings on SAD associated with fcc-Al are perfectly circular for the sputtered-film in contrast to the intermittent rings observed for the melt-spun ribbon. The specimens heated up to the second crystallization event showed different type of devitrification products. The remaining amorphous phase in the melt-spun ribbons transformed into a metastable hexagonal structure as predicted by in-situ XRD. Figure 4 (c) shows the rod like metastable hexagonal structure embedded in the remaining amorphous and nanocrystalline fcc-Al matrix. The sputtered-film, on the other hand, has transformed into an equiaxed metastable cubic structure as shown in Figure 4 (d). The corresponding diffraction patterns support the polycrystalline nature of the microstructure having ring patterns belonging to a metastable cubic and fcc-Al phases. 

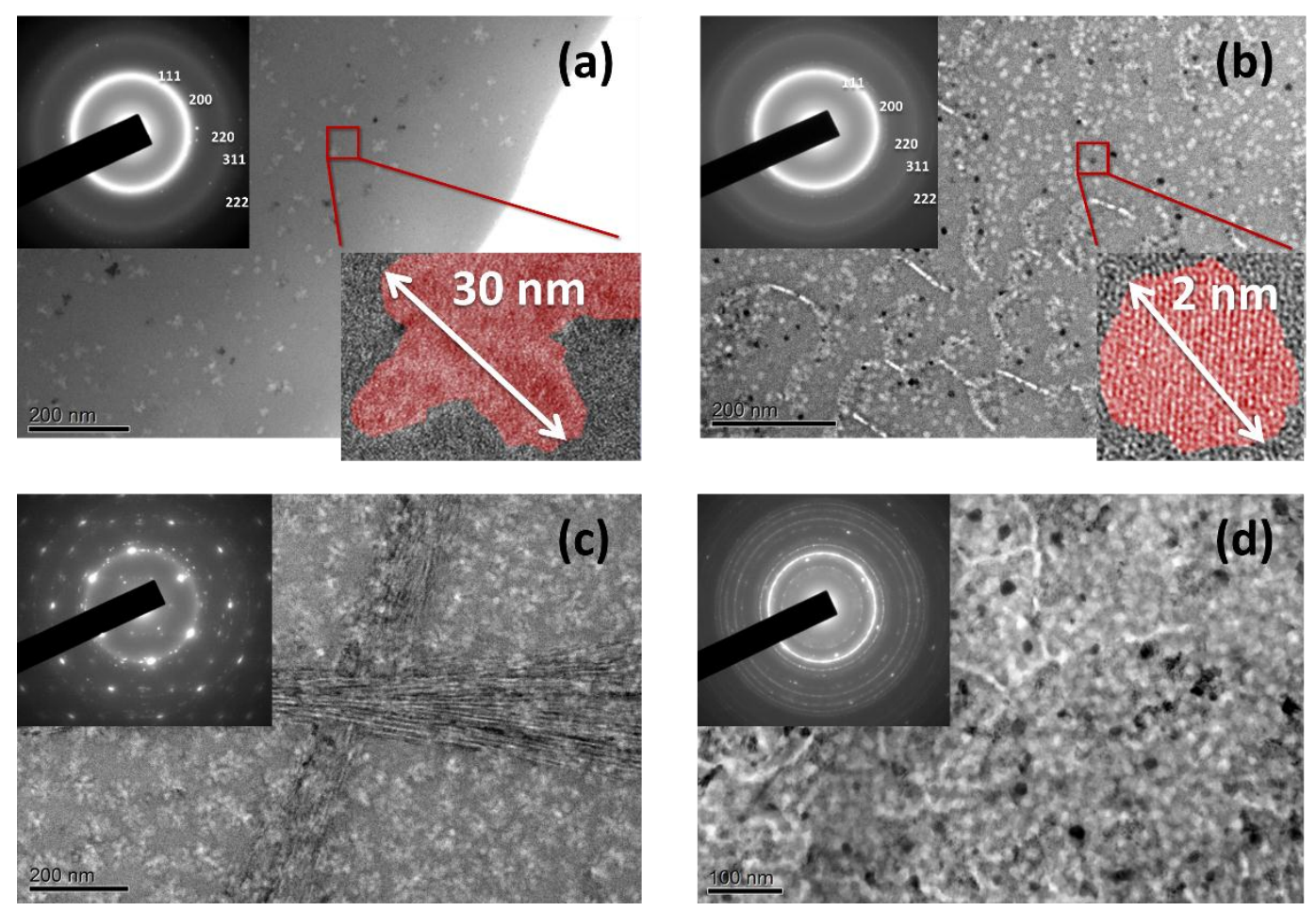

Figure 4. Bright-field TEM and selected area diffraction patterns (left insets) of (a) melt-spun ribbon quenched from $516 \mathrm{~K}$, (b) sputtered thin-film quenched from $505 \mathrm{~K}$, (c) melt-spun ribbon quenched from $536 \mathrm{~K}$, (d) sputtered thin-film quenched from $549 \mathrm{~K}$.

DSC, in-situ XRD, and TEM analyses evidently indicate a different devitrification pathway for amorphous the sputtered thin-films and the melt-spun ribbons that are of identical chemical compositions. Thus, the question is: why do two amorphous precursors having the same chemistry select different devitrification pathways? The XRD and in-situ TEM experiments showed that both amorphous phases initially crystalize to nanocrystalline fcc-Al. Subsequently, the ribbons exhibited a metastable hexagonal structure which quickly transforms into the metastable cubic phase. The residual amorphous phase in sputtered thinfilms, on the other hand, directly transforms into the metastable cubic structure. The differences in devitrification path should be attributed to the alteration of amorphous precursor. Although both specimens were found to be fully amorphous within the limitations of XRD, HRTEM, and SAD; the detailed investigations of pre-peak revealed that the differences between amorphous sputtered thin-film and melt-spun ribbon may be present in medium- 
range order length scale. Our previous studies on similar Al-RE systems showed that the liquid state of such systems is not chemically and topologically homogeneous as expected. Chemical separation reaching to $1 \mathrm{~nm}$ in size can be present in molten Al-RE alloys $[17,19]$. The separation observed in solid state should be inherited from the liquid state. During the quenching from liquid, these regions showed a subtle growth without compromising their non-crystalline nature and resulted in a solid amorphous phase which is chemically separated into Al-rich and Al-depleted regions. In our previous study, we called the Al-depleted regions "superatoms" to emphasize their ability of cross-linking in the amorphous matrix to form a network structure with regions of almost pure $\mathrm{Al}[14]$. The extra reflections in the total structure factors should be related to the Al-depleted regions of the divided matrix. Careful examination of the in-situ XRD patterns (Figure $3(a)$ ) revealed that the strong pre-peak observed in melt-spun ribbon evolves to the Bragg reflections at around $13 \mathrm{~nm}^{-1}$. Such a transformation is missing for the sputtered thin-films (Figure 3 (b)). We believe that the metastable hexagonal structure observed in the melt-spun specimen should be originated from the Al-depleted regions. The chemical and/or topological order in the Al-depleted regions makes this metastable hexagonal structure energetically favorable to form before the metastable cubic structure. The precursor of the amorphous thin-films is not a molten structure; it is vapor phase which is considerably more disordered compared to the liquid state and results in substantially higher cooling rates upon quenching. Therefore, it is quite plausible to anticipate a more chemically and topologically homogenous disordered structure with less extensive MRO as opposed to what is observed in melt-quenching. In general sense, this statement coheres well with our XRD observations: The width of the pre-peak is well broadened for the sputtered thin-films. The absence of metastable hexagonal structure formation during the devitrification confirms the lack of any significant separation in the solid 
state. However, the TEM analyses still indicate the nanocrystallization of fcc-Al at lower crystallization temperature with even higher number density $\left(\sim 10^{24} \mathrm{~m}^{-3}\right)$. This may be attributed to the existence of a much finer scaled network of Al rich regions in amorphous matrix that is formed by a nano-scale phase separation of main amorphous phase upon heating. As stated earlier, in the melt-spun ribbons, due to the strong interaction between Al and $\mathrm{Tb}$ atoms, the atomic arrangement in liquid state is already heterogeneous [13-15] and during solidification from melt, this heterogeneity is retained in the solid amorphous state and the structure consists of cross-linked "superatoms" and pure Al regions. However, due to the fact that the sputtered thin-films were deposited from vapor precursors, accompanied with the higher cooling rate, the solid amorphous phase may lack the chemical and topological heterogeneity that the melt-spun ribbons possess. Therefore, upon devitrification, before the commencement of the initial fcc-Al nanocrystallization, the amorphous matrix decomposes into Al-rich and Al-depleted regions to form a chemical and topological order similar to the melt-spun ribbon which has inherited its heterogeneity from liquid state. The nano-scale phase separation prior to fcc-Al crystallization also explains the size and morphology difference in fcc-Al nanocrystals devitrified from the amorphous matrix between the sputtered thin-film and the melt-spun ribbons. The matrix is divided into Al-rich and Aldepleted regions, and fcc-Al nucleates and grow from the Al-rich regions; as the nucleation of fcc-Al proceeds, nuclei of nanocrystals reject Tb atoms into the remaining Tb-rich amorphous phase. Due to the presence of a larger number density fcc-Al nucleation sites formed via nanoscale phase separation in the sputtered thin-film, the amount of Tb atoms that are expelled to the amorphous matrix becomes much higher as compared to that of melt-spun ribbons due to increased number of the nucleation sites. Thus, we can conclude that the soft impingement of Tb diffusion fields occurring in the sputtered thin-films is more aggressive in contrast to that 
of the melt-spun ribbons. This provides a reasonable explanation to the highly restricted growth of the fcc-Al nanocrystals within the sputtered thin-films. Moreover, the increased number of soft impingement events in the sputtered-thin films affects the morphology of the fcc-Al nanocrystals growing within the amorphous matrix. The shape of the nanocrystals in the sputtered thin-films become spherical to decrease their surface potential associated with the strain caused by the excess amount of Tb being rejected by the growing nanocrystals.

The presence of highly populated nanocrystals embedded in the amorphous matrix was previously reported to reduce the brittleness problem frequently encountered in metallic glass systems [23-25]. In this regard, the observed change in the morphology and the population of the fcc-Al nanocrystals in sputtered thin-films is highly encouraging. As stated before, by changing the cooling rate and the amorphization precursor, the morphology of the fcc-Al nanocrystals has changed from dendritic to spherical, together with two orders of magnitude increase in the population. These observations implies an enhanced mechanical behavior for the sputtered thin-films over melt-spun ribbons, as their ductility and hardness will increase with such microstructure. Implementation of a similar nanocrystalline/metallic glass composite structure would be promising in creating high strength and corrosion resistance materials for emerging surface engineering technologies.

\section{Conclusion}

The effects of altering the amorphous precursor on phase selection, nucleation and growth behavior in Al-Tb marginal glass forming alloy were comprehensively investigated by a combination of different thermal, electron and X-ray oriented characterization techniques. DSC and in-situ XRD results showed that the amorphous as-sputtered thin-films and asquenched ribbons of $\mathrm{Al}_{90} \mathrm{~Tb}_{10}$ alloys were observed to select different devitrification paths. 
TEM analyses revealed the existence of certain peculiarities in the evolving phases during the devitrification of the melt-spun ribbons and the sputtered thin-films. Notably, remarkable differences in the size and morphology for fcc-Al nanocrystals are observed. Moreover, the first intermetallic phase transformation following the fcc-Al nanocrystallization showed diversity between the melt-spun ribbons and the sputtered thin-films. Such differences can be related to degree of topological and chemical order within the amorphous precursors; the ribbons that were quenched from the liquid state already possess chemical separation up to some degree as opposed to the sputtered thin-films that were quenched from a more disordered phase (vapor) at a higher cooling rate. The fcc-Al and metastable hexagonal phases observed during the devitrification of the melt-spun ribbons are inherited from the Al-rich and the Al-depleted regions. On the other hand, for the devitrification of the sputtered thin-films, the metastable hexagonal intermetallic phase is bypassed and a metastable cubic phase is formed following the fcc-Al nanocrystallization. This can be attributed to the homogeneity of the structural disorder persisting in the amorphous matrix of the as-sputtered films which upon thermal devitrification, was observed to lead to a nano-scale phase separation of the Alrich and the Al-depleted regions, before the fcc-Al nanocrystallization. This prominently fine scaled regions result in a higher amount of Tb atoms that are pushed to the amorphous matrix during heating which induces highly restricted growth of fcc-Al with almost spherical nanocrystal/amorphous interface in the sputtered thin-films.

\section{Acknowledgement}

This work was financially supported by METU under the contact number of BAP-07-02-2012101 and partially supported by the Scientific and Technological Research Council of Turkey (TUBITAK) under Grant No. 113M346. The high-energy in-situ X-ray experiments were 
performed at the BLO4 - MSPD beamline of the ALBA Synchrotron Facility and financially supported by CALIPSO program. Inmaculada Peral and Oriol Vallcorba are acknowledged for their valuable help in HEXRD experiments. Work at Ames Laboratory, sample synthesis, was supported by the US Department of Energy, Basic Energy Sciences, Division of Materials Science and Engineering, under Contract No. DE-AC02-07CH11358.

\section{REFERENCES}

[1] A. Inoue, H.M. Kimura, K. Sasamori, T. Masumoto, Ultrahigh Strength of Rapidly Solidified Al96-xCr3Ce1Cox ( $\mathrm{x}=1,1.5$ and 2\%) Alloys Containing an Icosahedral Phase as a Main Component, Mater. Trans. JIM. 35 (1994) 85-94. doi:10.2320/matertrans1989.35.85.

[2] R.F. Cochrane, P. Schumacher, A.L. Greer, Crystallization of amorphous Al85Ni10Ce5 alloy, Mater. Sci. Eng. A. 133 (1991) 367-370. doi:10.1016/0921-5093(91)90089-6.

[3] E. Axinte, Metallic glasses from "alchemy" to pure science: Present and future of design, processing and applications of glassy metals, Mater. Des. 35 (2012) 518-556. doi:10.1016/j.matdes.2011.09.028.

[4] A. Inoue, H. Kimura, Fabrications and mechanical properties of bulk amorphous, nanocrystalline, nanoquasicrystalline alloys in aluminum-based system, J. Light Met. 1 (2001) 31-41. doi:10.1016/S1471-5317(00)00004-3.

[5] A.L. Greer, Metallic Glasses, Science (80-. ). 267 (1995) 1947-1953. doi:10.1126/science.267.5206.1947.

[6] A. Inoue, K. Ohtera, A.-P. Tsai, T. Masumoto, New Amorphous Alloys with Good Ductility in Al-Y-M and Al-La-M (M=Fe, Co, Ni or Cu) Systems, Jpn. J. Appl. Phys. 27 (1988) L280-L282.

[7] Y. He, S.J. Poon, G.J. Shiflet, Synthesis and Properties of Metallic Glasses That Contain Aluminum, Sci. . 241 (1988) 1640-1642. doi:10.1126/science.241.4873.1640.

[8] J. Henao, A. Concustell, I. G.Cano, S. Dosta, N. Cinca, J.M. Guilemany, et al., Novel Albased metallic glass coatings by Cold Gas Spray, Mater. Des. 94 (2016) 253-261. doi:10.1016/j.matdes.2016.01.040.

[9] R.B. Pond, Metallic filaments and method of making same, (1958). http://www.google.com/patents/US2825108.

[10] C. Suryanarayana, A. Inoue, Bulk Metallic Glasses, CRC Press, Boca Raton, FL, 2011. 
doi:10.1201/9781420085976-1.

[11] J.C. Foley, D.R. Allen, J.H. Perepezko, Analysis of nanocrystal development in Al-Y-Fe and Al-Sm glasses, Scr. Mater. 35 (1996) 655-660. doi:10.1016/1359-6462(96)001960 .

[12] G. Wilde, H. Sieber, J.. Perepezko, Glass formation in Al-rich Al-Sm alloys during solid state processing at ambient temperature, J. Non. Cryst. Solids. 250-252 (1999) 621625. doi:10.1016/S0022-3093(99)00147-7.

[13] K.F. Kelton, T.K. Croat, A.K. Gangopadhyay, L.-Q. Xing, A.L. Greer, M. Weyland, et al., Mechanisms for nanocrystal formation in metallic glasses, J. Non. Cryst. Solids. 317 (2003) 71-77. doi:10.1016/S0022-3093(02)02004-5.

[14] Y.E. Kalay, I. Kalay, J. Hwang, P.M. Voyles, M.J. Kramer, Local chemical and topological order in Al-Tb and its role in controlling nanocrystal formation, Acta Mater. 60 (2012) 994-1003. doi:10.1016/j.actamat.2011.11.008.

[15] K.K. Sahu, N. a. Mauro, L. Longstreth-Spoor, D. Saha, Z. Nussinov, M.K. Miller, et al., Phase separation mediated devitrification of Al88Y7Fe5 glasses, Acta Mater. 58 (2010) 4199-4206. doi:10.1016/j.actamat.2010.04.011.

[16] P. Juhás, T. Davis, C.L. Farrow, S.J.L. Billinge, PDFgetX3: A rapid and highly automatable program for processing powder diffraction data into total scattering pair distribution functions, J. Appl. Crystallogr. 46 (2013) 560-566.

doi:10.1107/S0021889813005190.

[17] Y.E. Kalay, L.S. Chumbley, M.J. Kramer, I.E. Anderson, Local structure in marginal glass forming Al-Sm alloy, Intermetallics. 18 (2010) 1676-1682.

doi:10.1016/j.intermet.2010.05.005.

[18] F. Zhang, Y. Sun, Z. Ye, Y. Zhang, C.-Z. Wang, M.I. Mendelev, et al., Solute-solute correlations responsible for the prepeak in structure factors of undercooled Al-rich liquids: a molecular dynamics study, J. Phys. Condens. Matter. 27 (2015) 205701. doi:10.1088/0953-8984/27/20/205701.

[19] M. Ovun, M.J. Kramer, Y.E. Kalay, Structural modeling of liquid and amorphous Al91Tb9 by Monte Carlo simulations, J. Non. Cryst. Solids. 405 (2014) 27-32. doi:10.1016/j.jnoncrysol.2014.08.037.

[20] Y.E. Kalay, C. Yeager, L.S. Chumbley, M.J. Kramer, I.E. Anderson, Initial crystallization in a nanostructured Al-Sm rare earth alloy, J. Non. Cryst. Solids. 356 (2010) 1416-1424. doi:10.1016/j.jnoncrysol.2010.05.005.

[21] Y.E. Kalay, L.S. Chumbley, I.E. Anderson, Crystallization behavior in a highly driven marginal glass forming alloy, J. Non. Cryst. Solids. 354 (2008) 3040-3048. doi:10.1016/j.jnoncrysol.2007.12.006.

[22] D.R. Allen, J.C. Foley, J.H. Perepezko, Nanocrystal development during primary crystallization of amorphous alloys, Acta Mater. 46 (1998) 431-440. doi:10.1016/S1359-6454(97)00279-6. 
[23] T. Demirtaş, Y.E. Kalay, Kinetics of fcc-Al nanocrystallization in Al90Tb10 metallic glass, J. Non. Cryst. Solids. 378 (2013) 71-78. doi:10.1016/j.jnoncrysol.2013.06.020.

[24] C.R. Cao, D.W. Ding, D.Q. Zhao, E. Axinte, H.Y. Bai, W.H. \& Wang, Correlation between glass transition temperature and melting temperature in metallic glasses. Materials \& Design, 60 (2014) 576-579. doi.org/10.1016/j.matdes.2014.04.021

[25] L.Z. Zhao, R.J Xue, Z.G. Zhu, Z. Lu, E. Axinte, W.H. Wang \& H.Y. Bai, Evaluation of flow units and free volumes in metallic glasses. Journal of Applied Physics, 116(10) (2014) 103516.doi.org/10.1063/1.4895586 
$\mathrm{Al}_{90} \mathrm{~Tb}_{10}$

Metallic Glass

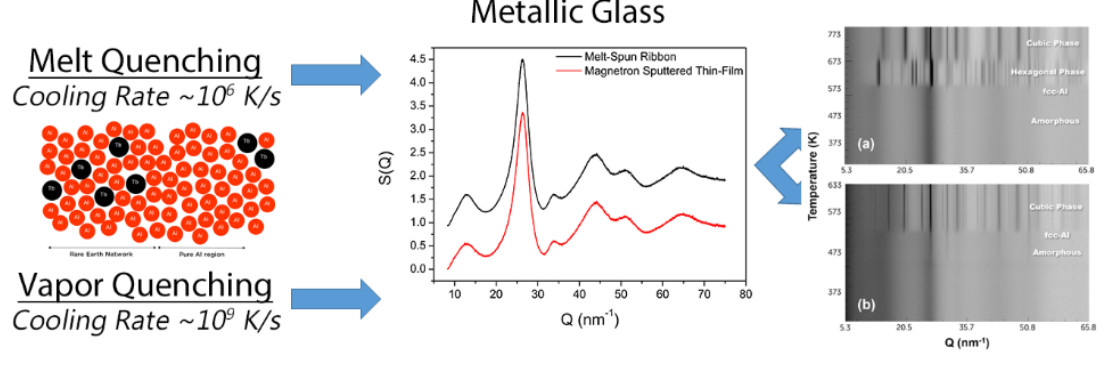

